\title{
Galactic Cosmic Ray induced absorbed dose rate in deep space - Accounting for detector size, shape, material, as well as for the solar modulation
}

\author{
Saša Banjac ${ }^{1, *}$, Lars Berger ${ }^{1}$, Sönke Burmeister ${ }^{1}$, Jingnan Guo ${ }^{1,2}$, Bernd Heber ${ }^{1}$, \\ Konstantin Herbst ${ }^{1}$, and Robert Wimmer-Schweingruber ${ }^{1}$ \\ ${ }^{1}$ IEAP, Kiel University, Christian-Albrechts-Platz 4, 24118 Kiel, Germany \\ ${ }^{2}$ School of Earth and Space Sciences, University of Science and Technology of China, Hefei, China
}

Received 4 December 2018 / Accepted 1 April 2019

\begin{abstract}
Depending on the radiation field, the absorbed dose rate can depend significantly upon the size of the detectors or the phantom used in the models. In deep space (interplanetary medium) the radiation field is on avarage dominated by Galactic Cosmic Ray (GCR) nuclei. Here, the deep space dose rate that a typical small silicon slab detector measures is compared to a larger phantom corresponding to an ICRU sphere with a $15 \mathrm{~cm}$ radius composed of water. To separate and understand respective effects from the composition, size and shape differences in the detectors, this comparison is implemented in several steps. For each phantom, the absorbed dose rate due to GCR nuclei up to $Z=28$, as a function of solar modulation conditions, is calculated.

The main components of the GCR flux are protons, followed by helium nuclei and electrons, with $Z>2$ nuclei accounting for approximately $1 \%$ of the total number of particles. Among the light nuclei with $Z>2$, most abundant ones are $\mathrm{C}, \mathrm{N}$ and $\mathrm{O}$. In this study, we use the GEANT4 model to calculate the absorbed dose (energy deposited as ionization, divided by mass) due to the GCR flux provided by the BadhwarO'Neill 2010 (BON-10) model. Furthermore, we investigate how the determined absorbed dose rate changes throughout the solar cycle by varying the GCR models from solar minimum to solar maximum conditions. The developed model is validated against the Cosmic Ray Telescope for the Effects of Radiation (CRaTER) microdosimeter measurements. In our current approach, we do not consider the effects of shielding, which will always be present under realistic scenarios.

A second goal of this study is to quantify the contribution of each $Z=1, \ldots, 28$ GCR nuclei to absorbed dose rate, in relation to the phantom characteristics. For each $Z$ we determine the most relevant energy range in the GCR spectra for absorbed dose rate estimations. Furthermore, we calculate a solar modulation dependent conversion factor to convert absorbed dose rate measured in silicon to absorbed dose rate in water. This information will improve our understanding of the radiation environment due to GCR in the near-Earth deep space and also benefit further modeling efforts by limiting the number and energy range of primary particle species that have to be considered.
\end{abstract}

Keywords: absorbed dose rate / radiation environment / cosmic ray / dosimetry / deep space

\section{List of acronyms}

CRaTER Cosmic Ray Telescope for the Effects of Radia- ICRP tion GCR Galactic Cosmic Ray

*Corresponding author: banjac@physik. uni-kiel.de
ICRP

ICRU

ISS
International Commission on Radiological Protection

International Commission on Radiation Units and Measurements

International Space Station 


$\begin{array}{ll}\text { LRO } & \text { Lunar Reconnaissance Orbiter } \\ \text { LIS } & \text { Local Interstellar Spectrum } \\ \text { LET } & \text { Linear Energy Transfer } \\ \text { MSL } & \text { Mars Science Laboratory } \\ \text { RAD } & \text { Radiation Assessment Detector } \\ \text { RWF } & \text { Radiation Weighting Factor } \\ \text { SDO } & \text { Solar Dynamics Observatory } \\ \text { SEP } & \text { Solar Energetic Particle } \\ \text { SRIM } & \text { Stopping and Range of Ions in Matter } \\ \text { TEM } & \text { Tissue Equivalent Material }\end{array}$

\section{Introduction}

The Galactic Cosmic Ray (GCR) flux is often approximated as a constant, isotropic radiation field which ionizes matter. On Earth's surface, the magnetosphere and atmosphere provide sufficient shielding against any potential radiation hazard to life that GCR might have. However, since in space the shielding provided to astronauts is limited (ranging between $1 \mathrm{~g} \mathrm{~cm}^{-2}$ and $100 \mathrm{~g} \mathrm{~cm}^{-2}$ of spacecraft material depth [e.g., Zeitlin et al., 2013] compared to $1100 \mathrm{~g} \mathrm{~cm}^{-2}$ of Earth's atmospheric depth), GCR induced radiation and its potential damage to manned space missions is an important concern. Therefore, a lot of effort has been put in to monitoring the radiation. Ideally, one is interested in determining the effective dose equivalent (for details see ICRP 123, Dietze et al., 2013), however this often requires experimental resources, in terms of mass, energy and volume, that are not always available. Thus, practically, monitoring is often realized by measuring the absorbed dose rate $\dot{D}$ using thin semiconductor detectors, performing a silicon-towater conversion and determining a mean quality factor $\bar{Q}$ based on a measurement of the Linear Energy Transfer (LET) spectrum $L$ and applying the corresponding quality function $\bar{Q}(L) \quad$ (ICRP 60, 1991). Regarding the silicon-to-water conversion, it is well known, that the measured silicon absorbed dose rate is lower than the absorbed dose rate in water would be due to the comparatively larger ionization potential of silicon. Thus, to convert the silicon dose, we multiply with a silicon to water conversion factor $\bar{f}_{\mathrm{Si}-\mathrm{H}_{2} \mathrm{O}}=1.2$ (e.g., Beaujean et al., 2002), originally based on ICRP 37 (Berger et al., 1984). In this work we will show that, in addition to the described effect of detector material, there is a significant non-trivial difference in the response of different detectors to radiation in relation to their size and shape. While the community was aware these factors play a role, up until now, they have not been quantified.

In our analysis we will compare typical semiconductor detectors to an ICRU sphere (ICRU 33, McNair, 1980), which is a $300 \mathrm{~mm}$ diameter sphere of ICRU 4-element tissue. The ICRU 4-element tissue (ICRU 33, McNair, 1980) has a density of $1 \mathrm{~g} \mathrm{~cm}^{-3}$, and a composition by mass of $76.2 \%$ oxygen, $11.1 \%$ carbon, $10.1 \%$ hydrogen, and $2.6 \%$ nitrogen. In our investigation, instead of the four-element Tissue Equivalent Material (TEM), we use water for the phantom composition. Our investigation of the impact of phantom size is a part of an ongoing investigation aiming to model the radiation environment within exoplanetary atmospheres. We have thus opted for more general results and chose water against the ICRU four- element tissue. However, when investigating the altitude depended absorbed dose rate in Earth's atmosphere, we have found that the results obtained with a water phantom and with a four-element TEM where within $2 \%$ of each other.

In space dosimetry, specifically, under circumstances where the experiment has to be designed under mass, energy and other constraints (e.g., on Mars), instead of a reference phantom, disk semiconductor detectors and slab semiconductor detectors are typically used (e.g., Lunar Reconnaissance Orbiter [LRO]/Cosmic Ray Telescope for the Effects of Radiation [CRaTER] microdosimeter experiment, or Mars Science Laborator [MSL]/Radiation Assessment Detector [RAD], which also uses a plastic scintillator with a composition similar to TEM). Absorbed dose rate alone is only one factor which determines the radiation burden to the astronauts, but for estimating radiation risks the radiobiological effectiveness of the different radiations play an important role for which a quality factor is defined (ICRP 60, 1991). For example, in the case of MSL/ RAD, the measured LET spectrum in silicon is converted to LET $\left(\mathrm{H}_{2} \mathrm{O}\right)$, and than a mean quality factor $\bar{Q}$ is calculated based on the quality function from ICRP 60 (1991). The measured absorbed dose rate $\dot{D}_{E}$ in the plastic scintillator detector (detector E) is multiplied with $\bar{Q}$ to obtain the dose equivalent rate $\dot{H}=\bar{Q} \dot{D}_{E}$.

We investigate in this paper the validity of the described silicon-to-water conversion factor and if/how it may change under different experiment setups including the detector size, shape, material and different GCR fluxes under different solar modulation conditions. Specifically, we would like to understand how the absorbed dose rate measured in a silicon detector compares to absorbed dose rate in an ICRU sphere of radius $r=15 \mathrm{~cm}$ composed of water. However, if we were to compare these two phantoms directly in our model, we could not attribute and quantify the contribution by each different effect including composition difference (water and silicon), shape difference (slab and sphere, disk) and size difference. In order to separate these effects, we have investigated the interaction of $Z=1, \ldots$, 28 GCR nuclei with seven detectors:

1. A silicon slab detector with thickness $H=350 \mu \mathrm{m}$,

2. a slab composed out of water of same dimensions,

3. a silicon disk detector with $H=350 \mu \mathrm{m}, r=520 \mu \mathrm{m}$,

4. a disk detector of equal dimensions composed out of water,

5. a spherical detector made out of silicon $(r=414 \mu \mathrm{m})$,

6. a spherical detector $(r=414 \mu \mathrm{m})$ composed out of water, and

7. a large International Commission on Radiation Units and Measurements (ICRU) water sphere with radius $r_{1}=15 \mathrm{~cm}$.

In the following text, these detectors will be designated as $\mathrm{Si}$ slab, $\mathrm{H}_{2} \mathrm{O}$ slab, Si disk, $\mathrm{H}_{2} \mathrm{O}$ disk, $\mathrm{Si}$ sphere, $\mathrm{H}_{2} \mathrm{O}$ sphere and ICRU (sphere). The thickness of the Si slab $(H=350 \mu \mathrm{m})$ has been chosen to correspond to a typical semiconductor detector employed in space dosimetry. We use this thickness as a reference value to determine for the other detector shapes the sizes so that a better separation of different effects is possible. Since GCRs are generally isotropic, unless within a highly nonuniform shielding environment, in all of our calculations we 
have sampled the initial polar direction angle from a cosine distribution from a sphere surrounding the detector. The mean path $\bar{x}$ through a slab (or a disk) of thickness $H$ can be determined analytically by calculating the mean polar angle (first moment) of the cosine distribution, i.e., by integrating $\int \theta \cdot \cos \theta \mathrm{d} \theta$ between $0^{\circ}$ and $90^{\circ} . \bar{x}$ is found to be equal to $\mathrm{H} / \mathrm{cos}$ $32.7^{\circ}=414 \mu \mathrm{m}$. Similarly, numerically we have determined that the mean path through a spherical volume of radius $r$ is equal to the radius $r$. That is, a sphere of radius $r=414 \mu \mathrm{m}$ would have the same mean path as a slab or a disk detector of thickness $H=350 \mu \mathrm{m}$, although the polar angle distribution of successful trajectories (hits) would be quite different between the detectors. The remaining physical quantities have been chosen in such a way that the sphere, disk and slab detectors have equal volume $V$ and, in cases of equal material, same density $\rho$ and mass $m$. Thus when comparing sphere, disc and slab detectors, we will be focusing on the differences in absorbed dose due to the differences of detector geometries. Alternatively, by considering detectors with the same shape, we can compare the effects attributed to the detector material. Detailed characteristics of the seven detectors can be found in Table 1. Moreover, we validate our method against actual deep space dose rate measurements obtained using the LRO/CRaTER microdosimeter experiment, of which the detector setup is also included as one of our models shown as CRaTER- $\mu$ in the table.

By comparing the absorbed GCR dose rate between detector (1) and (2), i.e., (3) and (4), and (5) and (6), we can attribute the absorbed dose rate difference to different compositions. The ratio of absorbed dose rate in water and in silicon of the same detector geometry corresponds to the previously introduced often used silicon to water conversion factor $f_{\mathrm{Si}-\mathrm{H}_{2} \mathrm{O}}$ (Sect. 1). By comparing (1)-(3) and (5) we could investigate how and why the resulting absorbed dose rates differ between slab-, discand spherically- shaped detectors. By comparing (1)-(5)/(6) and eventually to (7), we could understand how the detector size matters to the dose measurements of absorbed dose rate. The results presented hereafter will reveal a counter-intuitive, nontrivial relation between the described parameters and absorbed dose rate measurements.

The GCR composition has been the topic of numerous studies (Simpson, 1983; Tanabashi et al., 2018b). Due to a higher probability of fragmentation and generation of secondary particles via interaction with matter, simulating high energetic GCR nuclei ( $>\sim 1 \mathrm{GeV}$ ) with $Z>2$ is computationally very intensive. In the literature we find several studies investigating e.g., absorbed dose rate in the near-Earth space (Mrigakshi, 2013), or radiation within the atmospheres of Mars and Venus (Dartnell et al., 2015; Nordheim et al., 2015). More often than not, these results have been calculated by considering GCR nuclei with $Z$ up to 28 . Depending on the energy range and number of particles for the simulation, such simulations can require even months of processing time. Therefore, it would be of benefit to quantify how different GCR nuclei contribute to absorbed dose rate. That is, to reduce the number of species and the energy range down to the most relevant part in order to shorten the simulation time in future studies. We present here a detailed analysis of GCR-induced absorbed dose rate of different particle species under different solar modulation conditions.

Finally, we report such calculated modulation dependent factors for converting dose from silicon detectors to water material respectively for three different phantom geometries. In addition, we propose a novel silicon to ICRU sphere conversion factor which may be helpful for estimating biological radiation effects based on silicon dosimeter experiment in space.

\section{Model description}

The absorbed dose $D$ is defined to be the deposited ionization energy by charged particles per unit mass. Typically, we are interested in the absorbed dose rate $\dot{D}$, so that the absorbed dose is always specified per unit time. Using a detector of mass $m$, we can measure over a time interval $T$ the total ionization energy or, alternatively, we can sum up the contributions of the individual field particles:

$$
\dot{D}=\frac{D}{T}=\frac{E_{\text {total }}}{m \cdot T}=\frac{\sum_{i} E_{i}}{m \cdot T}
$$

How radiation interacts with the detector depends on the particle species and kinetic energy. The interaction of intermediate- $Z$ GCR components (heavy nuclei) at intermediate energies corresponding to $(0.1 \lesssim \beta \gamma \lesssim 1000)$ with matter is described to within a few percent by the Bethe equation (for the details thereof see Ch. 33 in Tanabashi et al., 2018a, and references therein). Here $\gamma$ and $\beta$ are the Lorentz-factor and the relativistic beta, respectively. The upper validity limit of $\beta \gamma \approx 1000$ corresponds to kinetic energies of $\approx 1 \mathrm{TeV}$ and $\approx 50 \mathrm{TeV}$, for protons and Iron nuclei, respectively. Above this energy, corrections like the density effect and others, become necessary.

The first step toward understanding how radiation interacts with a specific detector is to determine the average deposited ionization energy as a function of particle species $T$ and its kinetic energy $E_{i}$. The procedure described here is closely related to the one that has been extensively described and validated in the papers describing the absorbed dose calculation that has been implemented within the Atmospheric Radiation Interaction Simulator (AtRIS) particle transport code (Banjac et al., 2019). Using GEANT4 with the FTFP_BERT_HP physics list (Agostinelli et al., 2003; Allison et al., 2006, 2016; Ivanchenko et al., 2010), we have irradiated our detectors with an isotropic particle flux composed of GCR nuclei with $Z=1, \ldots, 28$. The particles were incident from half a hemisphere tangentially centered around a randomly chosen starting point on the detector surface and the direction polar angles were sampled using a cosine bias. The energies have been sampled from a flat spectrum over 650 logarithmically equidistant bins covering the range between $1 \mathrm{eV}$ and $10 \mathrm{TeV}$. The simulation considered hadronic and electromagnetic interactions, as well as models incorporating the decay of unstable particles.

Figure 1 shows the deposited energy $E_{d}$, as a function of kinetic energy $E_{i}$, in each of the eight different detectors, whereby the particle species are designated as shown in the figure legend. Each panel includes a reference line with a slope equal to 1 and corresponding to full absorption $E_{d}=E_{i}$ of the primary and all the ensuing secondaries. As we observe the graph of a particular GCR component, from lower toward higher energies, the graph first closely matches the reference line until it shifts downward as the energy becomes sufficient to penetrate the detector. For all detector setups, we observe 


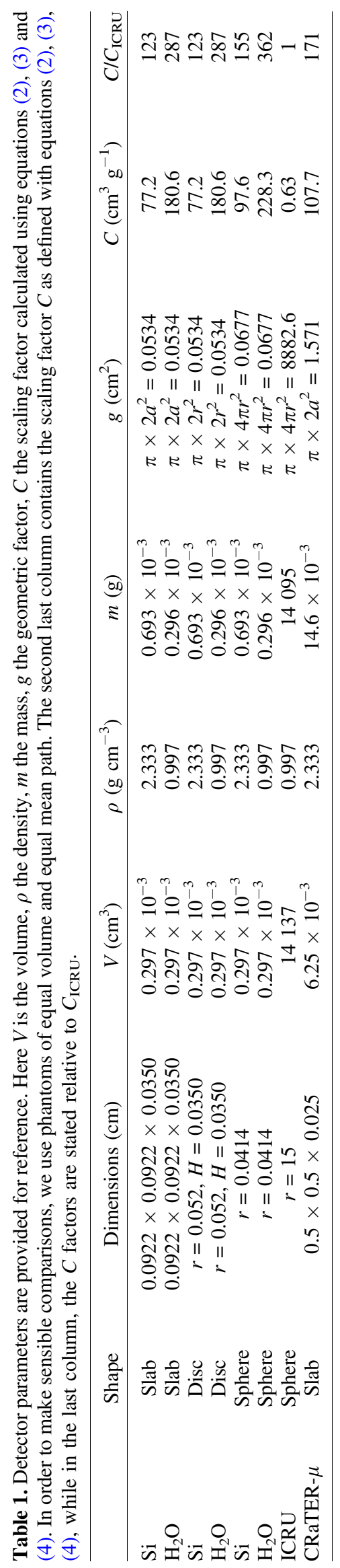

the expected difference between silicon and water. When comparing detectors of equal composition, the shape related differences are not extreme. However, the response of the large ICRU phantom is strikingly different with protons requiring as much as $100 \mathrm{MeV}$ to start penetrating. Meanwhile, the slab and disc detectors are penetrated by some protons with energies as low as $5 \mathrm{MeV}$ (or the ensuing secondaries). A $100 \mathrm{MeV}$ proton, however, deposits only $\approx 800 \mathrm{keV}$ to the $\mathrm{Si}$ sphere detector and $2 / 3$ of that to the Si disc and Si slab detectors. For all intents and purposes, the differences between the disc and slab detectors are exceedingly small. Their origin are boundary effects due to the fact that at the corners of the slab detector, the probability of sampling an unfavorable azimuth direction angle, that causes the particle to exit on the side, is higher than for the disc detector. Still, even in our unrealistic ratio between thickness and length, the described boundary effect is so minute that it can safely be neglected. Thus in the following discussion we will not consider the disc detectors any longer, since their response is emulated by the slab detectors.

Absorbed dose is ionization deposited in a given volume divided by its mass. Therefore, in order to compare detectors, we need to divide the deposited energy by the detector mass. Next, we need to take in to account that if the detectors were to be placed within the same radiation field, they would not get hit by a an equal number of particles. That is, we need to normalize the so determined absorbed dose rate to correspond to a given arbitrary flux. For a given differential flux $J$, which is typically expressed in units of $\mathrm{MeV}^{-1} \mathrm{~s}^{-1} \mathrm{sr}^{-1} \mathrm{~m}^{-2}$, the $g e$ ometric factor states the proportionality between the count rate (number of particles) and the flux $J$. For all discussed phantom geometries, the geometric factor is equal to $g=\pi A$, where $A$ is the surface of the phantom and $\pi$ is the factor obtained by integrating $J$ (Sullivan, 1971). In the third last column of Table 1 we have calculated the geometric factor for all detectors. For the slab and disc detectors, the surface has to be doubled, since they can be penetrated from both sides.

The two described normalization steps can be applied to the data shown in Figure 2 by multiplying it with:

$$
\begin{gathered}
C_{\text {Sphere }}=\frac{\pi \cdot A(r)}{m(r)}=\frac{\pi \cdot 4 \pi \cdot r^{2}}{\rho \cdot \frac{4}{3} \cdot \pi \cdot r^{3}}=\frac{3 \pi}{\rho \cdot r} \\
C_{\text {Slab }}=\frac{\pi \cdot A(r)}{m(r)}=\frac{\pi \cdot a^{2} \cdot 2}{\rho \cdot a^{2} \cdot H}=\frac{2 \pi}{\rho \cdot H} \\
C_{\text {Disc }}=\frac{\pi \cdot A(r)}{m(r)}=\frac{\pi \cdot \pi \cdot r^{2} \cdot 2}{\rho \cdot \pi \cdot r^{2} \cdot H}=\frac{2 \pi}{\rho \cdot H}
\end{gathered}
$$

For example, the factor $C$ is $0.63 \mathrm{~cm}^{2} \mathrm{~g}^{-1}$ and $97.6 \mathrm{~cm}^{2} \mathrm{~g}^{-1}$ for the ICRU phantom and the Si sphere detector, respectively. Thus the graphs corresponding to the $\mathrm{Si}$ sphere GCR response (green full line) are increased by a factor of $154.8 \times$, relative to those corresponding to the ICRU sphere (orange dashed lines). $C$ values and ratios $C / C_{\mathrm{ICRU}}$ comparing the scaling of the different detectors relative to the ICRU phantom, are found in the last two columns of Table 1 .

In Figure 2, we compare for protons, and $\mathrm{He}, \mathrm{O}$ and $\mathrm{Fe}$ GCR nuclei the response of the detectors after performing the described re-scaling. Each panel corresponds to the GCR nuclei 


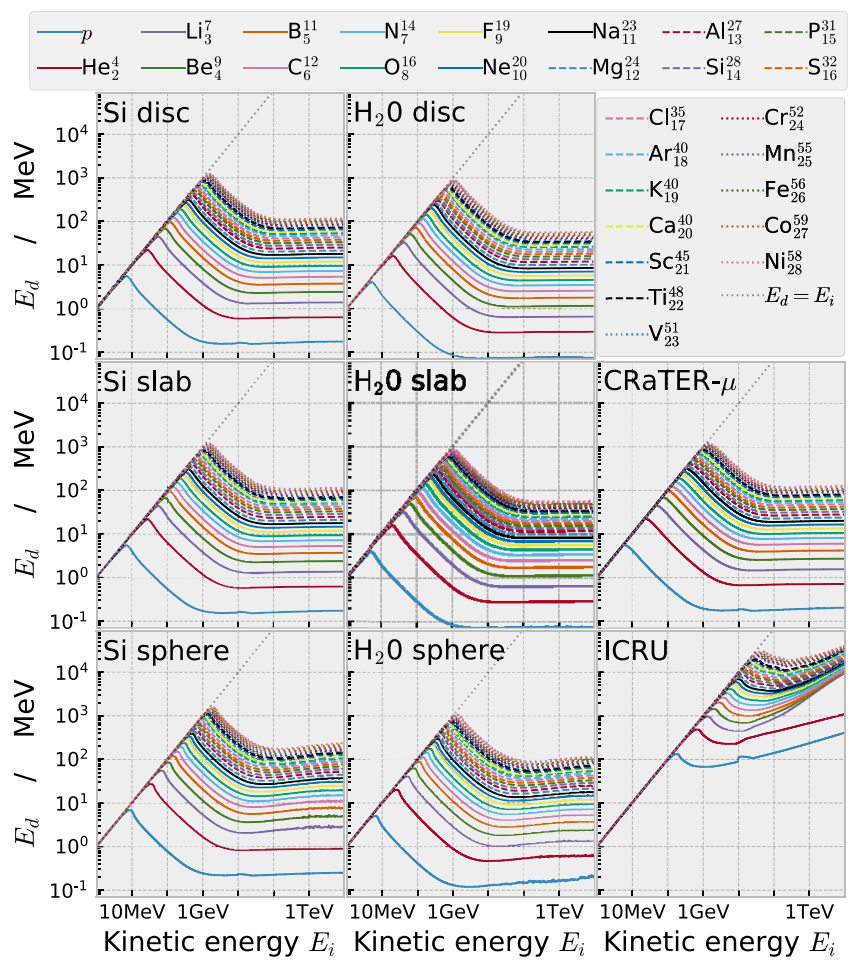

Fig. 1. Detector response to charged particles: the deposited energy $E_{D}$ is shown as a function of kinetic energy $E_{i}$. To each detector corresponds a single panel. For example the central panel is showing data for the $\mathrm{H}_{2} \mathrm{O}$ slab detector. The $\mathrm{Si}$ disc and $\mathrm{Si}$ slab detectors mimic typical semiconductor detectors used in space-related dosimetric experiments. The dotted line with slope 1 corresponds to full absorption. The different GCR nuclei are indicated with different colors and line styles, as shown in the legend.

indicated in the lower right corner. For all four nuclei, the large ICRU phantom suffers a higher absorbed dose than each of the detectors at the lower part of the energy spectrum. For protons, the ICRU water phantom begins to suffer a higher absorbed dose from $\approx 100 \mathrm{MeV}$ on-wards. For protons at $10 \mathrm{TeV}$ the difference between ICRU and the detectors is approximately two orders of magnitude. The responses of the detectors to alpha particles are very similar to their response to protons. However, as we irradiate the detectors with higher $Z$ nuclei, this crossing energy becomes higher, so that in the case of iron, the slab and sphere detectors suffers a higher dose up to $\approx 30 \mathrm{GeV}$. Furthermore, as $Z$ increases, the graphs of the ICRU water phantom at energies above $1 \mathrm{GeV}$ flatten-out, so that the difference between ICRU and the smaller detectors is reduced. An extended version of this analysis covering the first 28 GCR nuclei is presented in the Appendix (Fig. A1).

\subsection{GCR model and spectra}

The Badhwar-O'Neill 2010 (BON10, O'Neill, 2010a) model has been employed in the current study to generate primary GCR spectra of ion species with charge ranging from 1 to 28 . The BON10 spectra have been computed using the spherically symmetric Fokker-Planck equation, with the Local Interstellar Spectra (LIS) at the boundary of the heliosphere $(\sim 100$ $\mathrm{AU})$ as a boundary condition. The model uses an input parameter of the solar modulation $\phi$, also called the deceleration

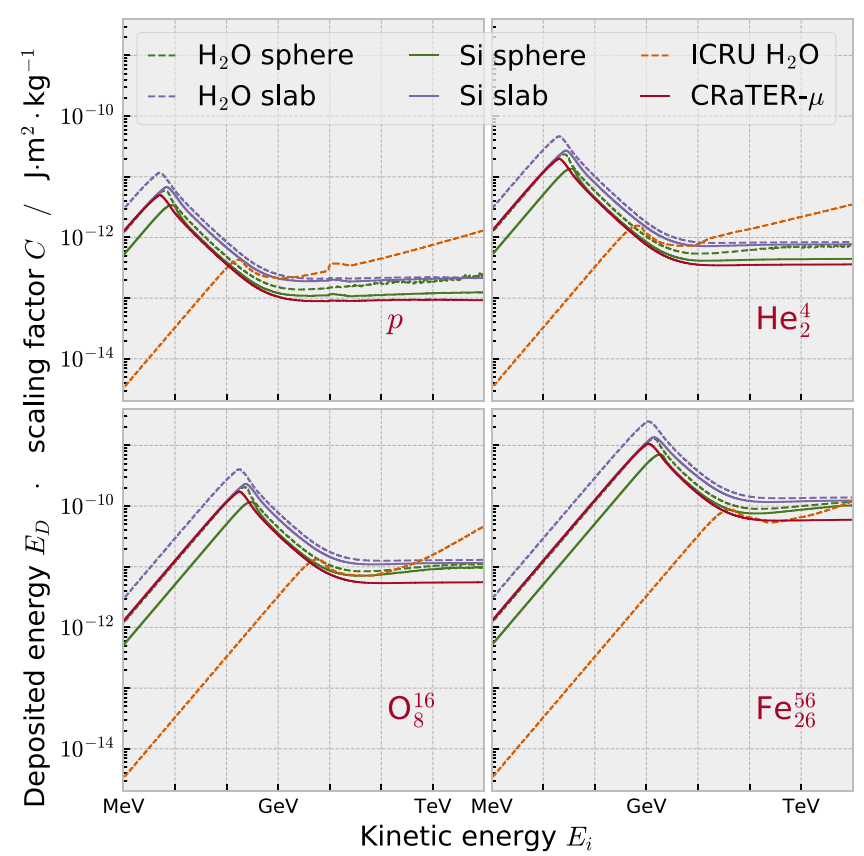

Fig. 2. Deposited energy multiplied by factor $C$ (Eqs. (2), (3) and (4)) takes in to account the fact that the phantoms have different masses and sizes. The analysis shown here is reduced to four GCR species: protons, and $\mathrm{He}, \mathrm{O}$ and $\mathrm{Fe}$ nuclei. After the re-scaling, we can compare the relative differences in absorbed dose rate that the phantoms would suffer if they were placed in to the same radiation field. We see that the the relation between phantom size, shape and measured dose has a non trivial dependence on particle type and energy. The calculated data indicates that the smaller slab and sphere phantoms suffers a higher absorbed dose for most particle types and energies.

parameter, which parameterize the solar activity and its modulation magnitude on the GCR particles. $\phi$ used in this study ranges from $400 \mathrm{MV}$ to $1500 \mathrm{MV}$, corresponding to solar minimum and solar maximum, respectively. Our investigation did not consider heavier nuclei $(Z>28)$ because (i) following Iron $(Z=26)$, the relative abundance of heavy GCR nuclei decreases rapidly (Alexeev et al., 2016), (ii) thus, their individual relative contribution is low (e.g., cobalt in Fig. 6 is below 0.1\%), and (iii) their simulation is computationally intensive.

In our model, for each GCR we used the energy range spanning from $1 \mathrm{MeV} \mathrm{nuc}^{-1}$ to $1 \mathrm{TeV}$ nuc ${ }^{-1}$. In Figure 3, we show the BON10 spectra for four GCR species selected out of the 28 species that have been used in our calculations. To each GCR nuclei corresponds a single panel, as is indicated in the upper right corner. Instead of plotting $J$ for a single value of the solar modulation parameter, we indicated with green shaded areas the whole range of possible values between solar minimum (upper, dashed black line, $\phi=400 \mathrm{MV}$ ) and solar maximum (lower, dotted black line, $\phi=1500 \mathrm{MV}$ ). By numerically integrating the differential fluxes, we get the total number of particles and with this we can compare the different GCR species according to their abundance. The most abundant GCR nuclei are protons, $\alpha$-particles, followed by Carbon, Oxygen and Nitrogen. By this measure, Iron is the 11th most abundant GCR nuclei, at least when performing the calculation for solar minimum periods $(\phi=400 \mathrm{MV})$. 


\section{Calculating and validating the absorbed dose rate}

The main result of our simulation is a function $E_{d}\left(Z, E_{i}\right)$, mapping kinetic energy $E_{i}$ and GCR nuclei $Z$ ( $Z$ designates the atomic number) to deposited energy $E_{D}$ (Fig. 1). Since we used 650 logarithmically equidistant bins spanning the energy range between $1 \mathrm{eV}$ and $10 \mathrm{TeV}$, the function is not continuous, but rather a step function. In the following, we designate with $E_{i}$ the center of the $i$ th kinetic energy bin. The GCR model described in Section 2.1 gives us the differential flux as a function of energy, particle type $Z$, and solar modulation parameter $\phi: J(Z, E, \phi)$. By integrating $J$ over the energy bin corresponding to $E_{i}$ and multiplying with the geometric factor $g$, we can calculate the number of particles $n\left(Z, E_{i}, \phi\right)$ hitting the detector. By performing a convolution over our energy binning of the two functions and by dividing with the mass of the phantom:

$$
\frac{E_{d}\left(Z, E_{i}, \phi\right) \times n\left(Z, E_{i}, \phi\right)}{\rho \cdot V}=: \dot{d}\left(Z, E_{i}, \phi\right)
$$

we get the absorbed dose rate as a function of particle species, kinetic energy and solar modulation parameter. $\dot{d}\left(Z, E_{i}, \phi\right)$ obviously depends on the choice of the detector and in Figure 4 we investigate for the four selected GCR species the differences between the detectors. Contrary to Figure 2, where we compared the detectors on the basis of an artificial flat spectrum, here we see how the absorbed dose rate seen by the different detectors differs in the case of a realistic spectrum. By integrating and summing up the 28 graphs, such as those shown in Figure 4, we get the net absorbed dose rate $\dot{D}$ seen by the detector:

$$
\dot{D}(\phi)=\sum_{Z \in[1, \ldots, 28]} \sum_{E_{i}} d\left(Z, E_{i}, \phi\right)
$$

\subsection{The impact of solar modulation}

Using a series of $\phi$ values and equation (6), we can calculate the the net absorbed dose rate in each detector as a function of $\phi$ (top panel, Fig. 5). The absorbed dose rate seen in the silicon slab (purple full line) is during solar minimum $\approx 1.43$ times higher than the absorbed dose rate seen in the ICRU water phantom. During solar maximum this factor decreases to $\approx 1.2$ (bottom panel, purple full line). However, following the standard procedure, the absorbed dose rate in silicon would be converted to absorbed dose rate in water by multiplying with a water conversion factor. In our analysis, this indirect approach was not necessary since we have also simulated a $\mathrm{H}_{2} \mathrm{O}$ slab detector. We see that the so determined absorbed dose rate in water using a slab detector overestimates the absorbed dose rate in the ICRU phantom by a factor of 2.1-1.7 (bottom panel, red full line). Instead of increasing the measured absorbed dose rate in silicon to get a dose rate meaningful for humans, one should decrease it with a factor corresponding to the $\mathrm{Si}$ slab/ICRU phantom ratio, which is also shown in the bottom panel using an orange full line. Finally, using our analysis we were able to derive an improved conversion factor as a function of the modulation parameter $\phi$, which is to be compared against factors typically found in the literature (green full line).

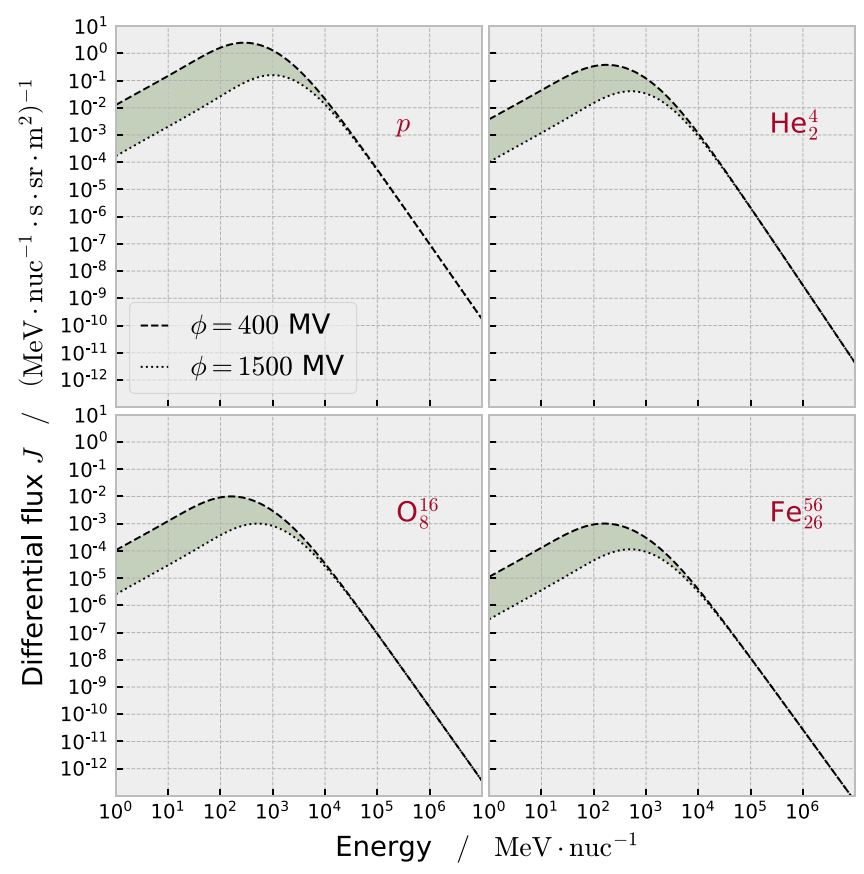

Fig. 3. BON10 spectra of protons (top left), helium nuclei (top right), oxygen nuclei (bottom left) and iron nuclei (bottom right), for two extreme cases of solar activity. During solar maximum (dotted black line, $\phi=1500 \mathrm{MeV}$ ) the flux of low energy particles decreases relative to periods of low solar activity (dashed black line), corresponding to a solar modulation parameter $\phi=400 \mathrm{MeV}$.

The conclusion is that the described difference in detector response to GCR nuclei results in an overestimation of the absorbed dose rate when performing dosimetric measurements employing thin semiconductor detectors. It is interesting to note that the overestimation factor is rather stable over the typical range of $\phi$ values: when comparing solar minimum to solar maximum, the difference is less than $20 \%$. Also, in the top panel of Figure 5, the red full line shows the absorbed dose rate dependence on deceleration parameter $\phi$ for the CRaTER- $\mu$ detector. The overestimation in this case is even more severe and this likely has to do with the microdosimeter having a thinner sensitive volume ratio (a thickness of $250 \mu \mathrm{m}$ compared to $350 \mu \mathrm{m}$ in the case of the slab detectors) and also having a larger surface to thickness ratio. We see that the detector thickness plays a crucial role for proper absorbed dose rate determination.

In order to investigate the contribution of different nuclei to the absorbed dose rate as a function of the modulation parameter $\phi$, we follow the procedure used to create Figure 5, by limiting the calculations to individual GCR nuclei and comparing to so obtained result to the net absorbed dose rate $\bar{D}$. We will compare how different GCR nuclei contribute to the calculated absorbed dose rate in the ICRU sphere and in the CRaTER- $\mu$ detector. Furthermore, a third case, accounting for the shielding surrounding the CRaTER microdosimeter (Mazur et al., 2011, 2013) is investigated. The results are shown in Figure 6, whereby the left panel corresponds to the ICRU phantom, while the middle and right panels are for the CRaTER- $\mu$ detector, without and with shielding, respectively. If we first concentrate on the ICRU phantom, we see that the protons and helium nuclei contribute the most to $\bar{D}$, with significant contributions due to carbon and oxygen. The figure includes several reference 


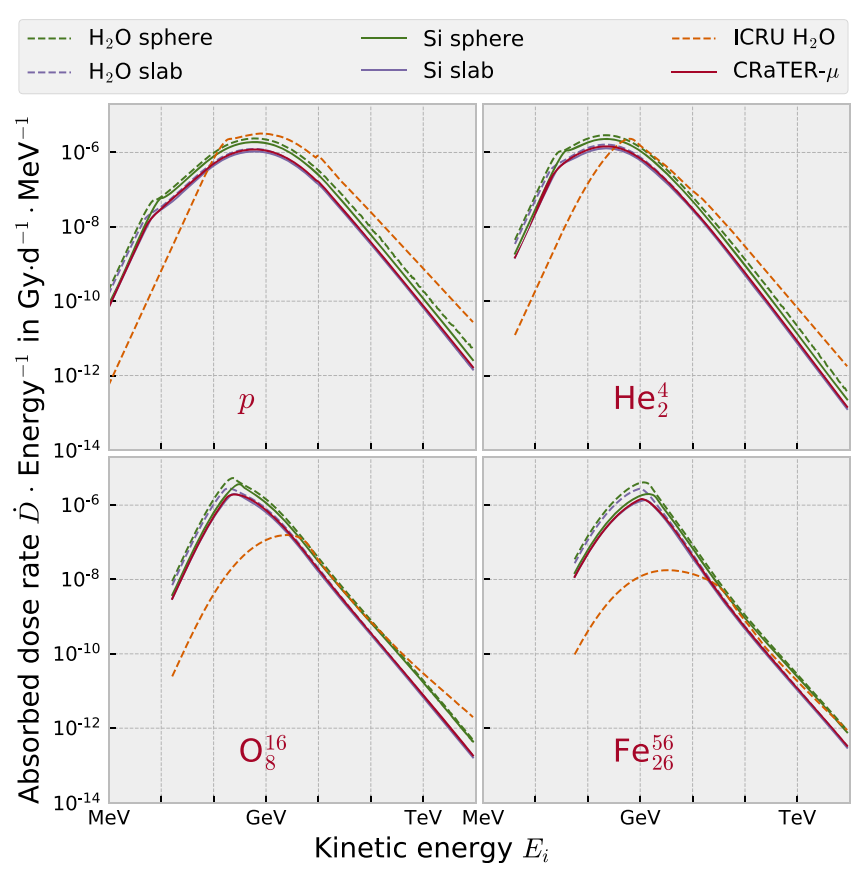

Fig. 4. Absorbed dose in $1 \mathrm{~s}$ as a function of energy during solar minimum $(\phi=400 \mathrm{MV})$ is shown here using four panels corresponding to the four selected GCR species, as indicated in the lower center of each panel. Full lines are used for silicon detectors, while dashed lines are used for $\mathrm{H}_{2} \mathrm{O}$ detectors. We observe that the smaller sphere and slab detectors suffer a higher dose at lower energies, while for the low- $Z$ nuclei above $1 \mathrm{GeV}$, the ICRU detector (phantom) suffers a slightly higher dose.

lines corresponding to a relative contribution of $10 \%, 1 \%$, etc. We can see that several species are just below the $1 \%$ line for solar minimum conditions, however as we move toward higher $\phi$ values, their importance increases so that at maximum $\phi$, Manganese, Nitrogen and Argon cross the $1 \%$ mark, while Iron, Beryllium and Neon almost reach it. These nuclei combined together can account for up to $6 \%$ of total absorbed dose rate at solar maximum. At minimum, their contribution is at most $3 \%$. The contribution of Carbon and Oxygen is between $4.5 \%$ and $8.5 \%$. Neglecting all other GCR nuclei would result in an error of at most $1 \%$ at solar maximum. If $7 \%$ precision is satisfactory, we can limit our analysis to protons, helium, carbon and oxygen. During solar minimum, the error of this approximation would reduce to below $3 \%$. As a further simplification, since the response of each phantom to carbon and oxygen is very similar (Figs. 1, 2 and 4), we could just simulate oxygen and include carbon by increasing the oxygen flux correspondingly. It is important to point out that we are analyzing the relative contribution. Thus an increase in the contribution of a particular GCR species is accompanied by a reduction in another one.

The middle and right panels of Figure 6 illustrate the severity of the discrepancy between a detector corresponding in size to humans (ICRU sphere) and thin semiconductor detectors. Here, the proton and helium contribution is reduced to less than $25 \%$ and we have more than a dozen of GCR species contributing more than $1 \%$ across all values of $\phi$. In particular, for the unshielded CRaTER- $\mu$ case (middle panel), iron contributes at solar maximum conditions to absorbed dose rate more than protons and helium.

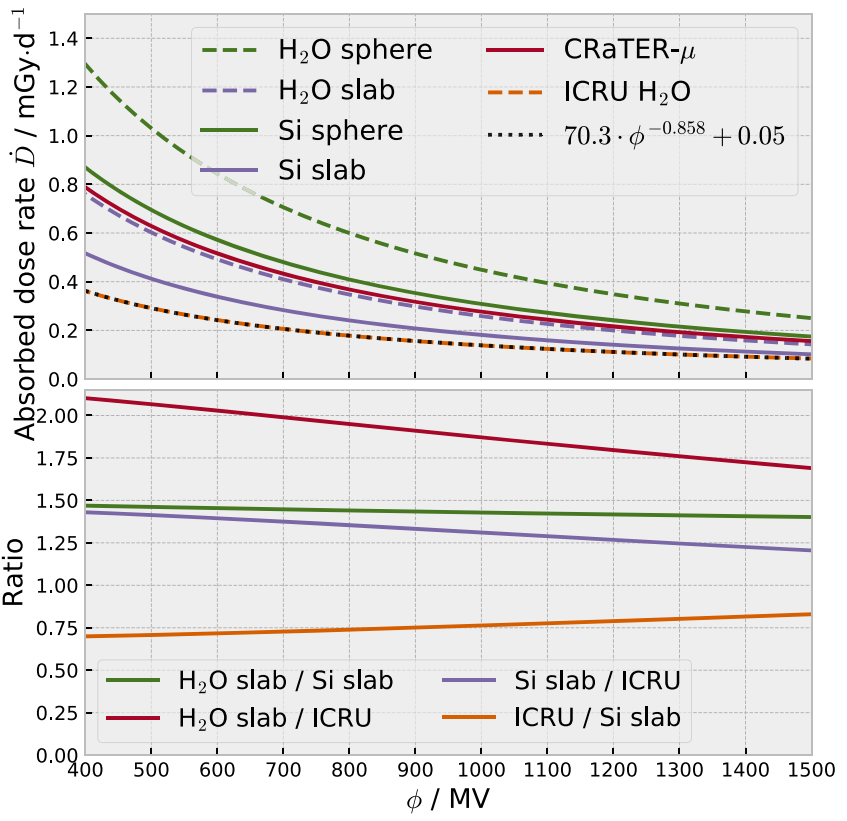

Fig. 5. Absorbed dose rate seen by the different detectors as a function of the solar modulation parameter has been obtained by calculating the absorbed dose rate using equation (6) for a series of $\phi$ values covering the range between $400 \mathrm{MV}$ and $1500 \mathrm{MV}$ using $10 \mathrm{MV}$ steps. All thin detectors overestimate the absorbed dose rate to the ICRU phantom (orange dashed line). These results indicate that measuring the absorbed dose rate using thin semiconductor detectors would result in an overestimation of the absorbed dose rate relative to the ICRU sphereradiological hazard to humans and that this overestimation increases as the detector thickness is reduced $($ CRaTER $-\mu)$. The dotted black line in the upper panel, overlapping the ICRU plot, represents the best fit in the least-squares sense.

This non-intuitive result can be understood by first considering Figure 3, which conveys that the iron flux has a maximum at $\approx 160 \mathrm{MeV}$ nuc $^{-1}$ during solar minimum and at $\approx 500 \mathrm{MeV}$ nuc $^{-1}$ during solar maximum. In general, for all GCR species, the maximum shifts toward higher energies during solar maximum, since the effect of solar modulation is more effective against lower energy particles. If we now consider Figure 1, we see that, at the corresponding kinetic energies (8.4 GeV and $28 \mathrm{GeV}$, respectively), the iron nuclei deposit a large portion of their kinetic energy within both the ICRU sphere and the CRaTER- $\mu$ detectors. The two detectors are able to completely stop $15 \mathrm{GeV}$ and $1.2 \mathrm{GeV}$ iron nuclei, respectively. However, since absorbed dose rate is ionization per unit mass, the resulting absorbed dose rate per single Iron particle is much lower in the ICRU sphere. This conclusion is supported by Figure 2, where the deposited energy is normalized against mass and the geometric factor of the detectors. We see that at energies below $30 \mathrm{GeV}$, the thin detectors suffer a significantly higher absorbed dose rate. Finally, the same is confirmed by the lower right panel of Figure 4.

Mostly due to their lower mass, the response of the thin detectors in terms of absorbed dose rate is orders of magnitudes higher than the response of the ICRU sphere, and as we move toward higher $Z$ nuclei, this response peak is shifted toward energy ranges in which the high $Z$ nuclei are significantly 


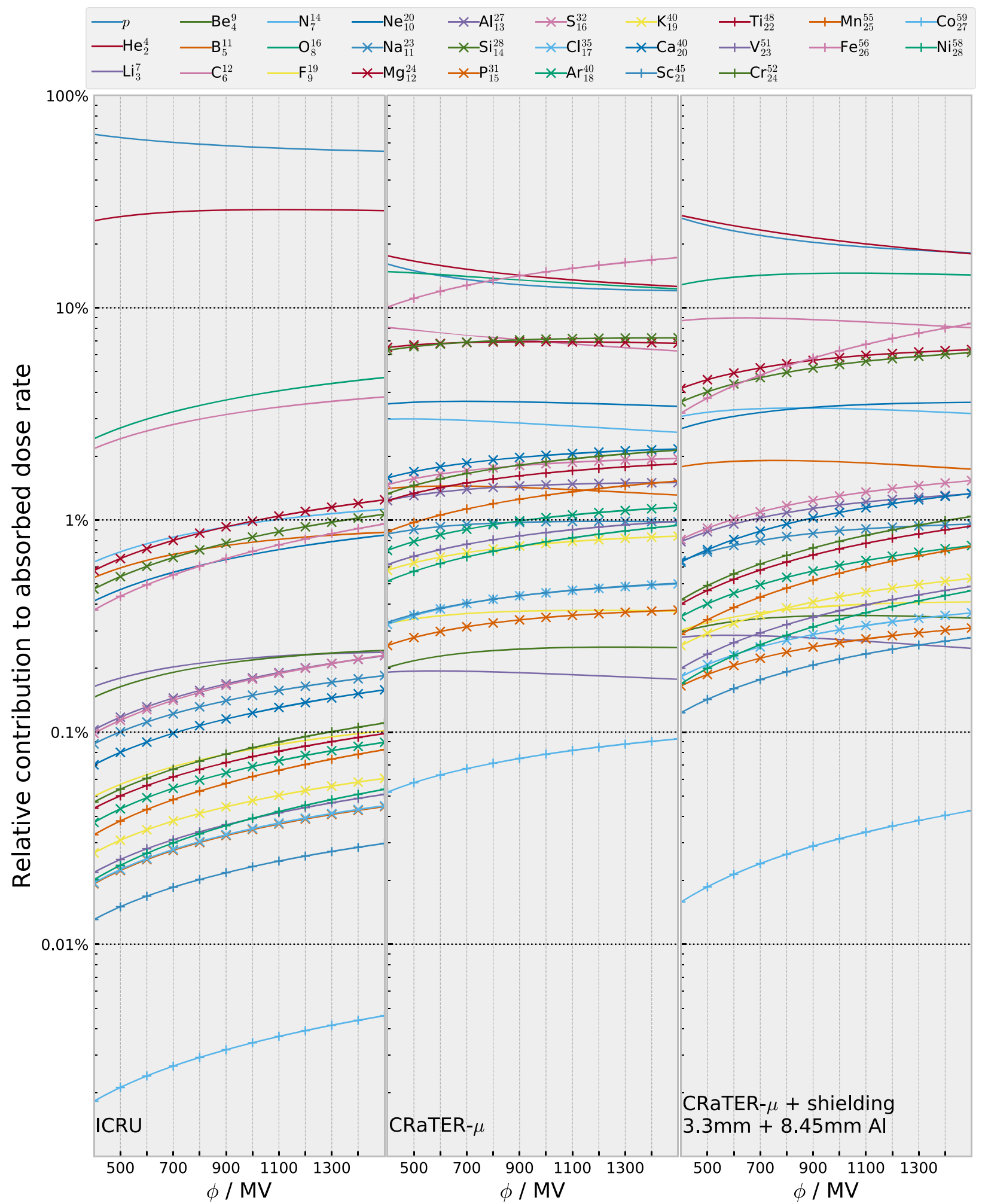

Fig. 6. The relative contribution to absorbed dose rate of different GCR nuclei has been calculated as a function of the solar modulation parameter $\phi$, that is shown on the $x$-axis. The different GCR nuclei are indicated using different line markers and colors (see legend). For the ICRU phantom, the most contributing GCR species are protons, followed by helium, oxygen and carbon nuclei. The contribution of $Z>1$ GCR species increases with $\phi$ at the cost of the proton contribution. The CRaTER- $\mu$ detector experiences a significantly higher contribution due to $Z>2$ GCR nuclei, however, with shielding, this effect is somewhat suppressed. 
abundant, and in the case of iron we observe a "resonance" effect. However, if we include shielding in our analysis, the situation becomes less critical (right panel of Fig. 6). Nevertheless, the calculated contributions to the shielded CRaTER- $\mu$ detector are still not similar to those of an ICRU sphere (left panel). However, if we consider the fact that the dose equivalent is often calculated by scaling the deposited energy using a quality factor which is $10 \times$ higher for $Z>1$ ions than for protons, we see that as a consequence of this higher response of thin detectors to $Z>1$ GCR nuclei, the dose equivalent could be greatly overestimated.

\subsection{Generating a time series and comparing with data}

By using the BON10 model together with the $\phi$ data that has been published in O'Neill (2010b, Fig. 6 therein), we can recreate the absorbed dose rate due to GCR as would have been seen by the detectors over the period between 1970 and October 2010. However, the time series of $\phi$ values has to be extended in order to make comparison against more recent measurements possible. Similar to the BON10 model, which is based on the spherically symmetric Fokker-Planck equation, the force-field solution by Usoskin et al. (2011) can be used to recreate the proton and helium spectra at 1 AU. In Figure 7, we compare the BON10 $\phi$ values to the $\phi_{\mathrm{PP}}$ values by Gieseler et al. (2017), which represent an improvement of the model by Usoskin et al. (2011). The Gieseler et al. (2017) $\phi$ (full black) and BON10 $\phi$ values (dotted red) are compared in the top panel. Even though the two sets of $\phi$ 's correspond to different models, they both are derived from measurements of solar activity and the agreement between the two is rather good, both in shape, as well as in magnitude. Since we have shown that the calculated dose rate is rather insensitive to the actual value of $\phi$ (Fig. 5) and since the topic being discussed focuses on discussing measurement principles, rather than predicting future absorbed dose rates, we will use the $\phi_{\mathrm{PP}}$ by Gieseler et al. (2017) to extend the time series from 2010 up to 2018. In the bottom panel, the reconstructed absorbed dose rates for the $\mathrm{H}_{2} \mathrm{O}$ sphere, $\mathrm{Si}$ sphere, $\mathrm{H}_{2} \mathrm{O}$ slab, $\mathrm{Si}$ slab and ICRU $\mathrm{H}_{2} \mathrm{O}$ sphere are shown. The shaded areas around the graphs correspond to our model uncertainty and are calculated by allowing for a $20 \%$ error in the value of $\phi$.

\subsection{Comparison against the CRaTER microdosimeter}

The CRaTER is a dosimetric experiment (Spence et al., 2010) aboard the LRO, which has been orbiting the Moon since 2009. In this work we will compare simulation results to measurements obtained using the CRaTER microdosimeter, which uses a single $5 \mathrm{~mm} \times 5 \mathrm{~mm} \times 0.25 \mathrm{~mm}$ semiconductor detector. Absorbed dose rate measurement performed by LRO/ CRaTER microdosimeter (Mazur et al., 2011, 2013) for the time period between 2009 and 2017 are shown with an orange full line in Figure 8. The shown CRaTER microdosimeter results are part of the original data product, which corrects to compensate for different instrumental effects (e.g., the data is corrected to compensate for the fact that the Moon is blocking a large portion of the solid angle, and that this correction varies with the

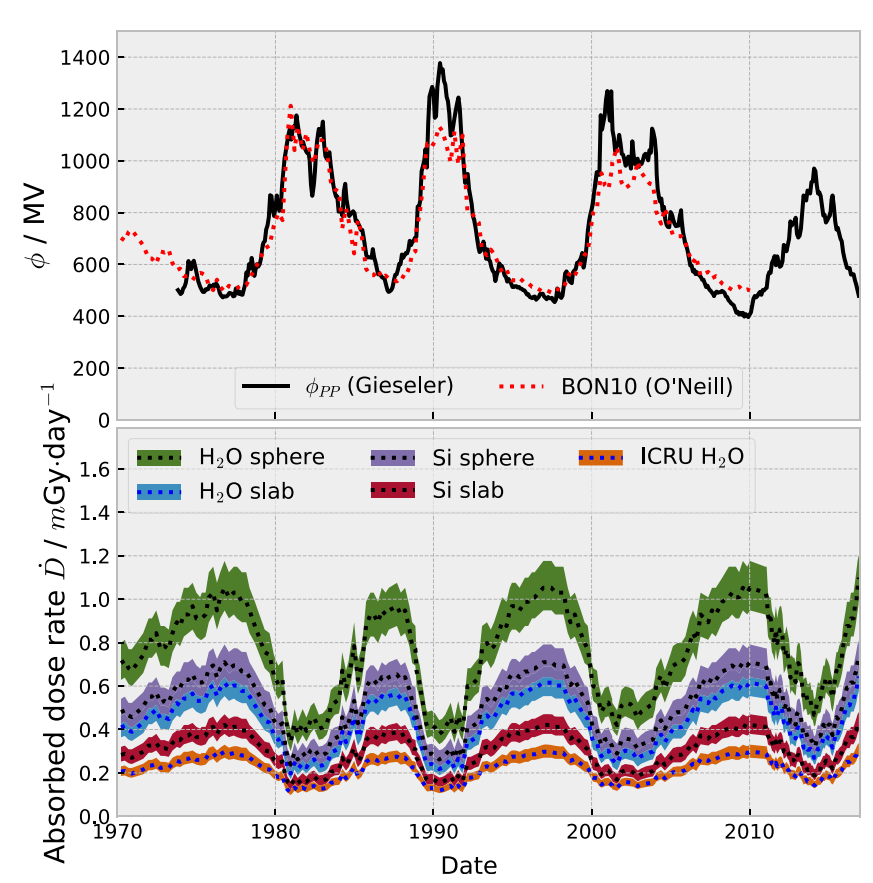

Fig. 7. The upper panel shows the $\phi$ parameter values by O'Neill (2010b) and Gieseler et al. (2017). In the lower panel, we show the time profile of absorbed dose rate due to GCR calculated with equation (6) while using the BON10 $\phi$ values for the period before 2010 and the Gieseler et al. (2017) thereafter. The absorbed dose rates seen by the different detectors are indicated with colors. The shaded area around the curves correspond to a $20 \%$ uncertainty in the value of $\phi$. For more details see text.

altitude of the LRO orbit). As such, it corresponds to the Deep space absorbed dose rate in water that one should measure in near-Earth space. In order to make the results comparable to our silicon phantom, we have performed a re-scaling using the silicon-to-water conversion factor of 1.33 , which is provided within the data product. To make visual comparison straightforward, we applied a simple filter to remove some of the Solar Energetic Particle (SEP) contamination. Please note that the shown time period covers only a small portion at the end of the time series shown in Figure 7.

Simulation results are shown using a red dashed line, with the shaded area corresponding to a $20 \%$ uncertainty in the $\phi$ value. To account for the shielding around the microdosimeter $\left(0.89 \mathrm{~g} \mathrm{~cm}^{-2}\right)$ on one side and $2.28 \mathrm{~g} \mathrm{~cm}^{-2}$ on the other side (Mazur et al., 2011), an additional CRaTER detector with $3.3 \mathrm{~mm}$ aluminum shielding on one side and $8.45 \mathrm{~mm}$ on the other side has been simulated. Results thereof are shown using a blue dotted line. While the unshielded results recreate the general trend of the CRaTER measurements, they are higher, in particular during periods of low solar activity $(\phi \approx 500 \mathrm{MV})$. The agreement between simulation and measurement during solar maximum is better, with the two time series being within $20 \%$ of each-others uncertainty range. The higher error during solar minimum can be well explained by the fact that the microdosimeter is housed within the casing of CRaTER and that this casing provides shielding which is particularly efficient at blocking the GCR nuclei with lower energies. Thus, during solar minimum, when there is a higher number of lower energy 
particles (Fig. 3), many of those get slowed or blocked within the casing of the instrument.

However, if we take the shielding around the instrument in to account, we get outstanding agreement between simulation (blue dotted line) and measurement. In our model, this has been implemented by sandwiching the detector between two aluminum slabs with thicknesses corresponding to the published information on the shielding around the CRaTER microdosimeter. After applying this improvement, we can even observe that the measurement closely matches the variability of the GCR flux which is included in the simulation results. To summarize, the response of the thin slab and disc detectors to GCR nuclei differ greatly from the response of an ICRU sphere. As a consequence, utilizing such dosimeters to measure the dose rate results in an overestimation of the actual dose rate that the ICRU water phantom would suffer. Consequent multiplication of dose rates to convert from silicon to water makes this overestimation even worse.

The investigated impact of shielding on the microdosimeter results motivated us to try to modify the shielding to better match the absorbed dose rate time profile seen by the ICRU sphere. We have found out that even the small microdosimeter with dimensions equal to $5 \mathrm{~mm} \times 5 \mathrm{~mm} \times 0.25 \mathrm{~mm}$ can be modified to measure the dose rate that an ICRU phantom would suffer. To achieve this, we have investigated how different shielding thickness modify the absorbed dose rate measurements and we found out, that if the CRaTER microdosimeter is enclosed in to $5 \mathrm{~mm}$ thick aluminum shielding, it's response is modified to match the response of the ICRU sphere. The top panel of Figure 9 shows the absorbed dose rate dependence on the modulation parameter $\phi$ for the ICRU sphere (full black) and the CRaTER microdosimeter with shielding (dotted red) and without (full orange) the $5 \mathrm{~mm}$ thick shielding. The full green line shows the CRaTER dose rate without shielding converted to water by multiplying with 1.33 . The bottom panel compares the measurement to this modified version of CRaTER. Also shown are two additional version with $3.3 \mathrm{~mm}$ and $8.45 \mathrm{~mm}$ casing, which show how the situation changes if less or more shielding is used. It is pure luck that the instrument's casing provided similar shielding, so that the total response of the CRaTER microdosimeter + instrument casing was close to the response that an ICRU sphere would have had. Considering the small size of the microdosimeter, this modification would further increase the mass of the dosimeter by only $35 \mathrm{~g}$, which corresponds to a small fraction of the mass of an ICRU sphere $(\approx 14 \mathrm{~kg})$. However, this approximation has to be used with caution, since its validity would not hold if the dosimeter would be placed in a realistic environment (i.e., a spacecraft with some shielding). It is useful only to evaluate the absorbed dose to an ICRU sphere due to GCR nuclei in a scenario with no shielding and thus presents limited value for the radiation protection community. The presented results are relevant since they hint toward the possibility of looking for a small detector system having a well defined response relative to a more realistic phantom, thus enabling realistic estimates of the radiation hazard using a small sensitive mass.

\subsection{Conversion factors for the 28 GCR species}

In Figure 5 we have published new conversion factors for slab detectors composed of silicon. Thanks to the flexibility of our approach we can calculate similar conversion factors

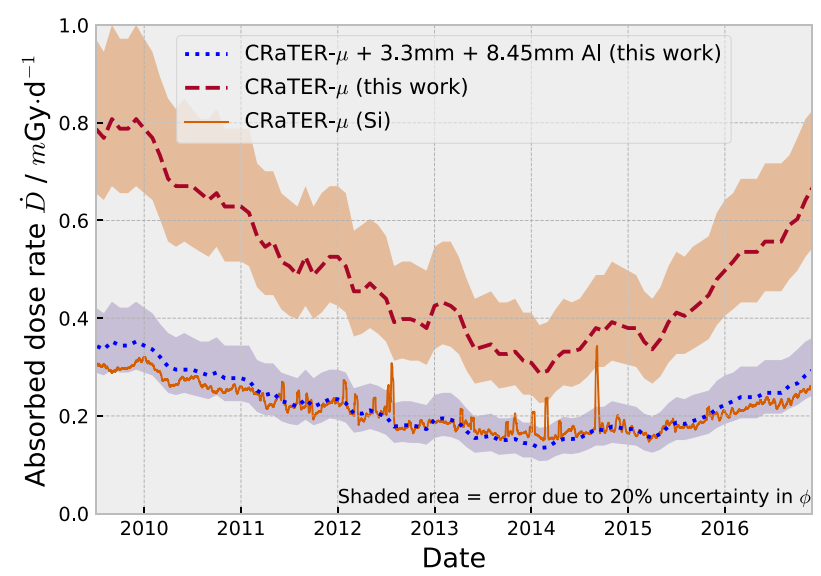

Fig. 8. To compare against CRaTER measurements, which are shown using a orange full line, we used two CRaTER-like phantoms: one without any shielding (red dashed line) and one with shielding corresponding to the actual shielding surrounding the CRaTER microdosimeter (blue dotted line). The agreement between measurements and the shielded CRaTER phantom is outstanding, so much that one can even interpret the variations in the measurements to be a consequence of the modelled variations in the GCR flux. The time series shown here corresponds to a small portion of the time series shown in Figure 7.

for all 28 GCR species. The factors for converting the absorbed dose rate in a silicon slab to absorbed dose rate in a water slab are shown in Figure 10. We observe a range of values between 1.2 (for protons) and 1.92 (for iron). Furthermore, our net conversion factor, indicated in Figure 10 with a dotted black line, has been calculated by taking in to account the differences in abundances of the different GCR nuclei. It decreases with increasing solar activity from $f_{\mathrm{Si}-\mathrm{H}_{2} \mathrm{O}}(\phi=400 \mathrm{MV})=1.49$ to $f_{\mathrm{Si}-\mathrm{H}_{2} \mathrm{O}}(\phi=400 \mathrm{MV})=1.43$. Figure $\mathrm{A} 4$, which can be found in the appendix, makes a similar comparison between the Si slab detector and the ICRU sphere. It gives an additional perspective of the importance of $Z>2$ GCR nuclei to the determination of absorbed dose rate within the thin phantoms. Please note, that in line with our other findings, these results are valid for the specific geometry that we are considering, i.e., a slab of $350 \mu \mathrm{m}$ thickness.

Benton et al. (2010) have investigated the silicon-to-water LET conversion and have published data giving the ratio $\mathrm{LET}_{\infty} \mathrm{H}_{2} \mathrm{O} / \mathrm{LET}_{\infty} \mathrm{Si}$ as a function of energy nuc ${ }^{-1}$ for several GCR species (H, He, C, O, Mg, Si, Ar and Fe) over the energy range extending from $1 \mathrm{MeV}$ nuc $^{-1}$ to $2 \mathrm{GeV}$ nuc $^{-1}$. Generally speaking, our comparison was not performed in terms of LET, but rather, in terms of absorbed dose rate. Furthermore, our comparison is based on a convolution with a realistic GCR spectrum, while theirs is performed in terms of single particles. However, since in this specific case we are comparing a water slab to a silicon slab, and since we can assume that the particles had a similar mean path through the detector body, we may compare qualitatively our findings with the findings of Benton et al. (2010), whose results indicate that the $\log \left(\mathrm{LET}_{\infty} \mathrm{H}_{2} \mathrm{O}\right)=-0.2902+1.025 \log \left(\mathrm{LET}_{\infty} \mathrm{Si}\right)$ and that there the $Z$-dependence converges at energies above $55 \mathrm{MeV} \mathrm{nuc}^{-1}$. At $1 \mathrm{MeV}$ nuc $^{-1}$, their published conversion 


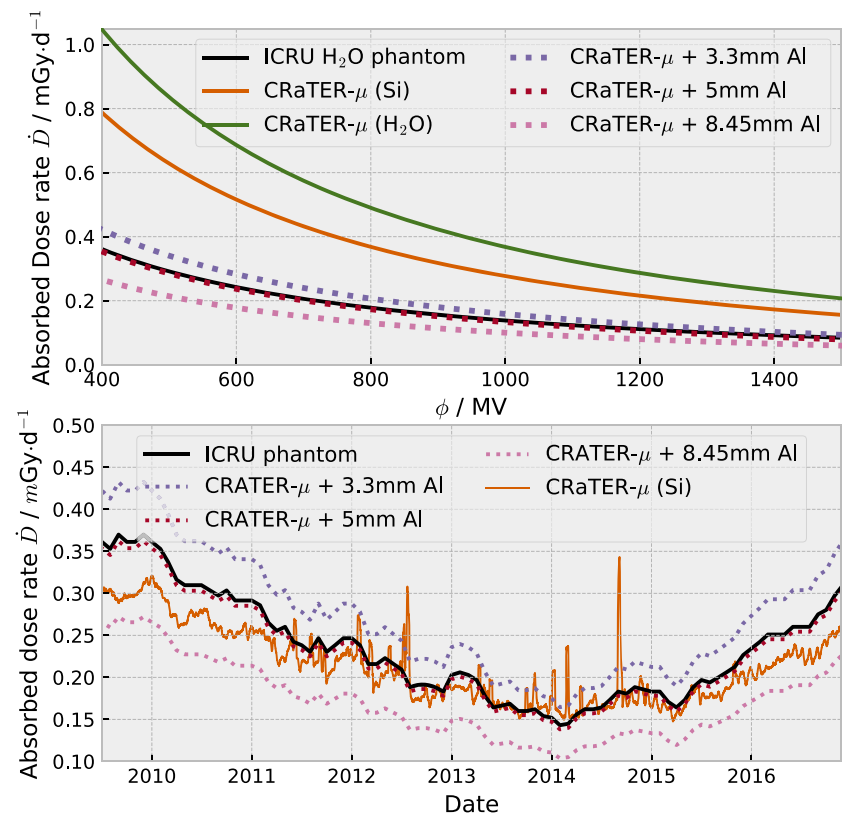

Fig. 9. Modifying the CRaTER- $\mu$ microdosimeter by adding a $5 \mathrm{~mm}$ thick aluminum casing emulates the ICRU sphere. The top panel shows the absorbed dose rate dependence on the modulation parameter $\phi$ in the range between $400 \mathrm{MV}$ and $1500 \mathrm{MV}$. Two additional configurations corresponding to $3.3 \mathrm{~mm}$ and $8.45 \mathrm{~mm}$ of aluminium shielding are shown to present the general trend. The bottom panel compares the absorbed dose rate that an ICRU sphere would suffer to the measurement and to the absorbed dose rate in the modified microdosimeter. Measuring absorbed dose rate in silicon using such a modified microdosimeter is sufficient to know the absorbed dose rate that a full ICRU water phantom would suffer in open space.

factors are highest: $\operatorname{LET}_{\infty} \mathrm{H}_{2} \mathrm{O} / \mathrm{LET}_{\infty} \mathrm{Si}$ (proton) $=0.665$, while $\mathrm{LET}_{\infty} \mathrm{H}_{2} \mathrm{O} / \mathrm{LET}_{\infty} \mathrm{Si}$ (iron) $=0.715$, corresponding to a constant factor of 1.33 and 1.43 , for protons and iron nuclei, respectively. Their data indicates that higher $Z$ particles have a larger conversion factor and that with increasing energy nuc ${ }^{-1}$, the conversion factor decreases. While the general trend behind these conclusions is in agreement with our findings, our results deliver considerably higher conversion factors, especially during solar-minimum conditions, where the lower energy GCR nuclei are more abundant. This difference is something that may well follow from the described dependence of absorbed dose rate upon the size and geometry of the detector. Furthermore, please note that data shown in Figure 4 shows that (i) the energies above $2 \mathrm{GeV} \mathrm{nuc}^{-1}$ cannot be ignored, at least in a radiation field corresponding to deep space, and (ii) that they do not support the conclusion of Benton et al. (2010) that the $\mathrm{LET}_{\infty} \mathrm{H}_{2} \mathrm{O} / \mathrm{LET}_{\infty} \mathrm{Si}$ ratio converges at energies above $55 \mathrm{MeV} n u c^{-1}$.

\section{Summary, conclusion and outlook}

In order to quantify the radiation hazard to humans in space, we need to be able to accurately measure the absorbed dose rate, which is the deposited ionization energy per unit time, divided

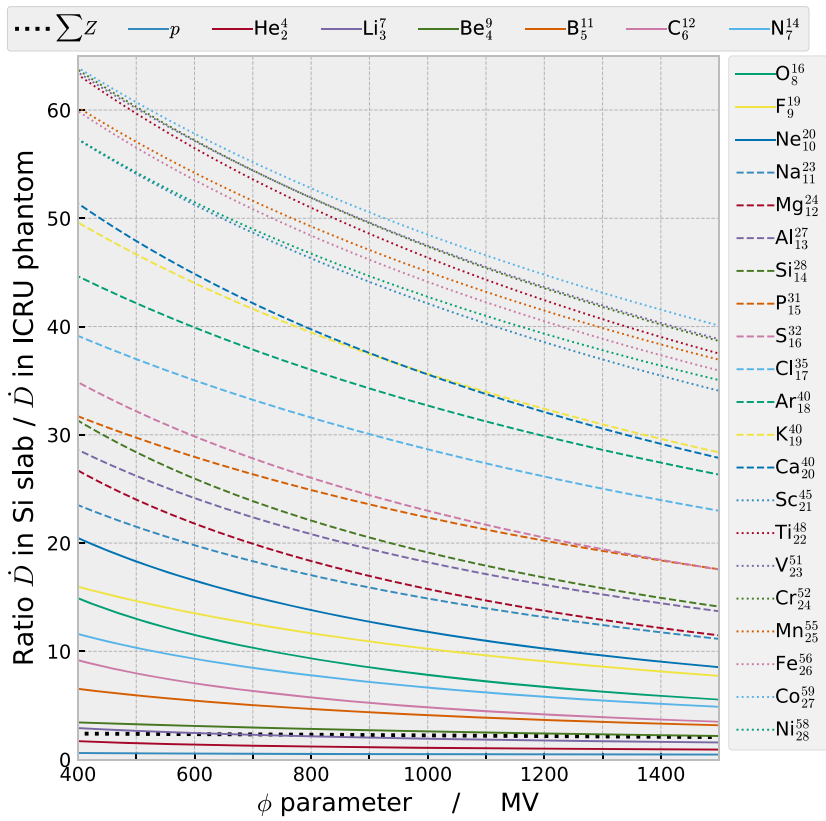

Fig. 10. Conversion factor between $\mathrm{Si}$ and $\mathrm{H}_{2} \mathrm{O}$ for the slab phantoms, as a function of the $\phi$ parameter and atomic number $Z$. The dotted black line indicates the net conversion factor.

with mass. In this investigation, we considered how the size, the shape and the material of the detector influences the determination of the absorbed dose rate. We have quantified (Fig. 5) how measurement techniques employing semiconductor detectors overestimate the absorbed dose due to GCR by a factor that is greater than two for all levels of solar activity in an environment with no or very little shielding. The trend shown in Figure 4 clearly indicates that a similar overestimation would be observed when measuring dose rates caused by SEP events. The validity of our results is supported by the outstanding agreement that has been observed when comparing our model to the CRaTER microdosimeter measurements (Fig. 8). While we have used a single GCR model in our investigation, nothing indicates that our conclusion would significantly change if another GCR model were to be used, since the differences between the models are minor and since the determined overestimation factor is relatively stable relative to small changes in the $\phi$ parameter. The main take-away message is that the detector geometry plays a decisive impact on the outcome of absorbed dose rate measurement.

Our investigation concentrated on quantifying the detector difference in terms of absorbed dose rate. For radiation risk estimates the effective dose equivalent instead would have to be determined. For such investigations depth dose measurements are necessary, which can be achieved by using phantoms mimicking a human. Several such phantom experiments were flown aboard the International Space Station (ISS), like e.g., Matroshka-1 (MTR-1) and Matroshka-2 (MTR-2) (e.g., Reitz \& Berger, 2006; Reitz et al., 2009). In addition, our results are relevant for space experiments where constraints do not allow for the use of human phantoms, since typically in such cases the dose equivalent is calculated by multiplying the measured absorbed dose with a mean quality factor that is obtained from a separate measurement of the LET spectrum based on: 


$$
Q(L)=\left\{\begin{array}{cc}
1.0, & \text { for } L<10 \mathrm{keV} / \mu \mathrm{m} \\
0.32 L-2.2, & \text { for } 10 \mathrm{keV} / \mu \mathrm{m} \leq L \leq 100 \mathrm{keV} / \mu \mathrm{m} \\
300 /\left(L^{12}\right), & \text { for } L \geq 100 \mathrm{keV} / \mu \mathrm{m}
\end{array}\right\}
$$

Our results have a considerable impact for the calculation of the dose equivalent. We plan to better quantify the errors playing a role in this calculation which are related to differences in detector size, shape and composition. Furthermore, to increase the practical relevance of our results, we will consider realistic shielding scenarios.

Acknowledgements. Our work has been supported by the German Research Foundation (DFG) under the project "The influence of Cosmic Rays on Exoplanetary Atmospheric Biosignatures". We would like to thank M. Sinnhuber and V. Schmidt (Karlsruhe Institute of Technology), as well as H. Rauer, L. Grennfell and M. Scheucher from the Technical University of Berlin. J.G. is partly supported by the Key Research Program of the Chinese Academy of Sciences under grant no. XDPB11. The editor thanks two anonymous referees for their assistance in evaluating this paper.

\section{References}

Agostinelli S, Allison J, Amako KA, Apostolakis J, Araujo H, et al. 2003. GEANT4 - a simulation toolkit. Nucl Instrum Methods Phys Res, A Accel Spectrom Detect Assoc Equip 506(3): 250-303.

Alexeev V, Bagulya A, Chernyavsky M, Gippius A, Goncharova L, et al. 2016. Charge spectrum of heavy and superheavy components of galactic cosmic rays: Results of the olimpiya experiment. Astrophys J 829(2): 120.

Allison J, Amako K, Apostolakis J, Arce P, Asai M, et al. 2016. Recent developments in Geant4. Nucl Instrum Methods Phys Res, A Accel Spectrom Detect Assoc Equip 835: 186-225.

Allison J, Amako K, Apostolakis JEA, Araujo H, Dubois PA, et al. 2006. Geant 4 developments and applications. IEEE Trans Nucl Sci 53(1): 270-278.

Banjac S, Herbst K, Heber B. 2019. The Atmospheric Radiation Interaction Simulator (AtRIS) - Description and validation. $J$ Geophys Res (Space Phys) 124(1): 50-67. DOI: 10.1029/ 2018JA026042.

Beaujean R, Kopp J, Burmeister S, Petersen F, Reitz G. 2002. Dosimetry inside MIR station using a silicon detector telescope (DOSTEL). Radiat Meas 35(5): 433-438.

Benton E, Benton E, Frank A. 2010. Conversion between different forms of LET. Radiat Meas 45(8): 957-959.

Berger M, Inokuti M, Anderson H, Bichsel H, Dennis J, Powers D, Seltzer S, Turner J. 1984. Report 37. J Int Comm Radiat Units Meas (2).

Dartnell LR, Nordheim TA, Patel MR, Mason JP, Coates AJ, Jones GH. 2015. Constraints on a potential aerial biosphere on Venus: I Cosmic rays. Icarus 257: 396-405.

Dietze G, Bartlett D, Cool D, Cucinotta F, Jia X, et al. 2013. Icrp publication 123: Assessment of radiation exposure of astronauts in space. Ann ICRP 42(4): 1-339.

Gieseler J, Heber B, Herbst K. 2017. An empirical modification of the force field approach to describe the modulation of galactic cosmic rays close to Earth in a broad range of rigidities. J Geophys Res (Space Phys) 122(11): 10,964-10,979.
ICRP Publication 60. 1991. 1990 Recommendations of the International Commission on Radiological Protection. Ann ICRP 21(1-3): $1-201$.

Ivanchenko V, Apostolakis J, Bagulya A, Abdelouahed H, Black R, et al. 2010. Recent improvements in Geant4 electromagnetic physics models and interfaces. In: 3th Monte Carlo Conference MC2010 vol. 2: 898-903.

Mazur J, Crain W, Looper M, Mabry D, Blake J, Case A, Golightly M, Kasper J, Spence HE. 2011. New measurements of total ionizing dose in the lunar environment. Space Weather 9(7).

Mazur JE, Zeitlin C, Schwadron N, Looper MD, Townsend LW, Blake JB, Spence H. 2013. Update on radiation dose from galactic and solar protons at the moon using the LRO/CRaTER microdosimeter. Space Weather 13(6): 363-364. DOI: 10.1002/ 2015SW001175.

McNair A. 1980. ICRU report 33 - Radiation Quantities and Units Pub: International Commission on Radiation Units and Measurements, Washington D.C. USA issued 15 April 1980, pp.25. J Labelled Compd Radiopharm 18(9): 1398-1398. DOI: 10.1002/ jlcr.2580180918.

Mrigakshi AI. 2013. Galactic cosmic ray exposure of humans in space - Influence of galactic cosmic ray models and shielding on dose calculations for low-Earth orbit and near-Earth interplanetary space. Ph.D. Thesis, University of Kiel, Germany. URL https:// elib.dlr.de/84322/.

Nordheim T, Dartnell L, Desorgher L, Coates AJ, Jones GH. 2015. Ionization of the Venusian atmosphere from solar and galactic cosmic rays. Icarus 245: 80-86.

O'Neill PM. 2010a. Badhwar-O'Neill 2010 galactic cosmic ray flux model - revised. IEEE Trans Nucl Sci 6(57): 3148-3153.

O'Neill PM. 2010b. Badhwar-O'Neill 2010 galactic cosmic ray flux model-Revised. IEEE Trans Nucl Sci 57(6): 3148-3153.

Reitz G, Berger T. 2006. The MATROSHKA facility-Dose determination during an EVA. Radiat Protect Dosim 120(1-4): 442445.

Reitz G, Berger T, Bilski P, Facius R, Hajek M, et al. 2009. Astronaut's organ doses inferred from measurements in a human phantom outside the International Space Station. Radiat Res 171 (2): 225-235.

Simpson JA. 1983. Elemental and isotopic composition of the galactic cosmic rays. Annu Rev Nucl Part Sci 33: 323-382. DOI: 10.1146/annurev.ns.33.120183.001543.

Spence HE, Case A, Golightly M, Heine T, Larsen B, et al. 2010. CRaTER: The cosmic ray telescope for the effects of radiation experiment on the lunar reconnaissance orbiter mission. Space Sci Rev 150(1-4): 243-284.

Sullivan J. 1971. Geometric factor and directional response of single and multi-element particle telescopes. Nucl Instrum Methods 95 (1): $5-11$.

Tanabashi M, Hagiwara K, Hikasa K, Nakamura K, Sumino Y, et al., (Particle Data Group). 2018a. Review of particle physics. Phys Rev D 98: 030,001. DOI: 10.1103/PhysRevD.98.030001.

Tanabashi M, Richardson P, Bettini A, Vogt A, Garren L, et al. 2018b. APS: Review of particle physics. Phys Rev D 98: 030,001.

Usoskin IG, Bazilevskaya GA, Kovaltsov GA. 2011. Solar modulation parameter for cosmic rays since 1936 reconstructed from ground-based neutron monitors and ionization chambers. $J$ Geophys Res (Space Phys) 116: A02104. DOI: 10.1029/ 2010JA016105.

Zeitlin C, Hassler D, Cucinotta F, Ehresmann B, WimmerSchweingruber R, et al. 2013. Measurements of energetic particle radiation in transit to Mars on the Mars Science Laboratory. Science 340(6136): 1080-1084. 


\section{Appendix}

To improve readability we have reduced within the main part of this publication the number of GCR nuclei to four: $\mathrm{H}$, $\mathrm{He}, \mathrm{O}$ and $\mathrm{Fe}$. In the appendix we provide an extended version of Figure 2 containing all 28 GCR nuclei (Fig. A1).

\section{Analyzing the contribution of GCR species to absorbed dose}

In this section we are going to investigate the energy dependence of $\dot{D}(\phi)$ for the two solar activity cases. In the six panel rows of Figure A2 we have calculated the normalized cumulative sums of $\dot{d}\left(Z, E_{i}, \phi\right)$ for all the 28 GCR nuclei under

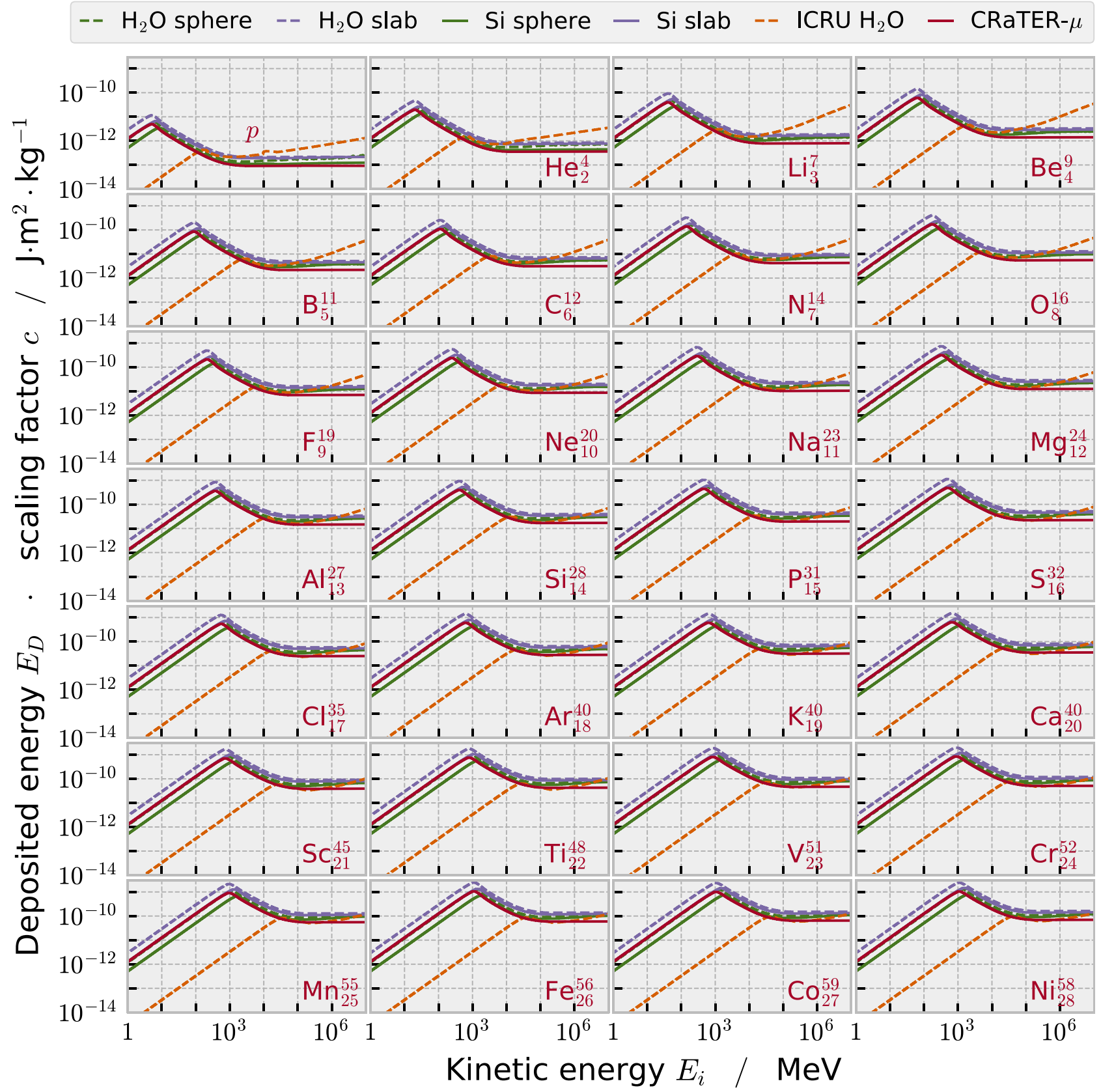

Fig. A1. Absorbed dose due to heavy particles multiplied by factor $C$ (Eq. (2)) takes in to account the fact that the phantoms have different masses and sizes. After doing so, we can compare the relative differences in absorbed dose rate that different phantoms would suffer if they were placed in to the same radiation field. Full lines correspond to silicon phantoms, while dashed lines are used for $\mathrm{H}_{2} \mathrm{O}$ phantoms. The different shapes and sizes are indicated with colors. We see that the the relation between phantom size and measured dose has a non trivial dependence on particle type and energy. The calculated data indicates that the silicon phantom suffers a higher absorbed dose than the ICRU phantom for most particle types and energies. 


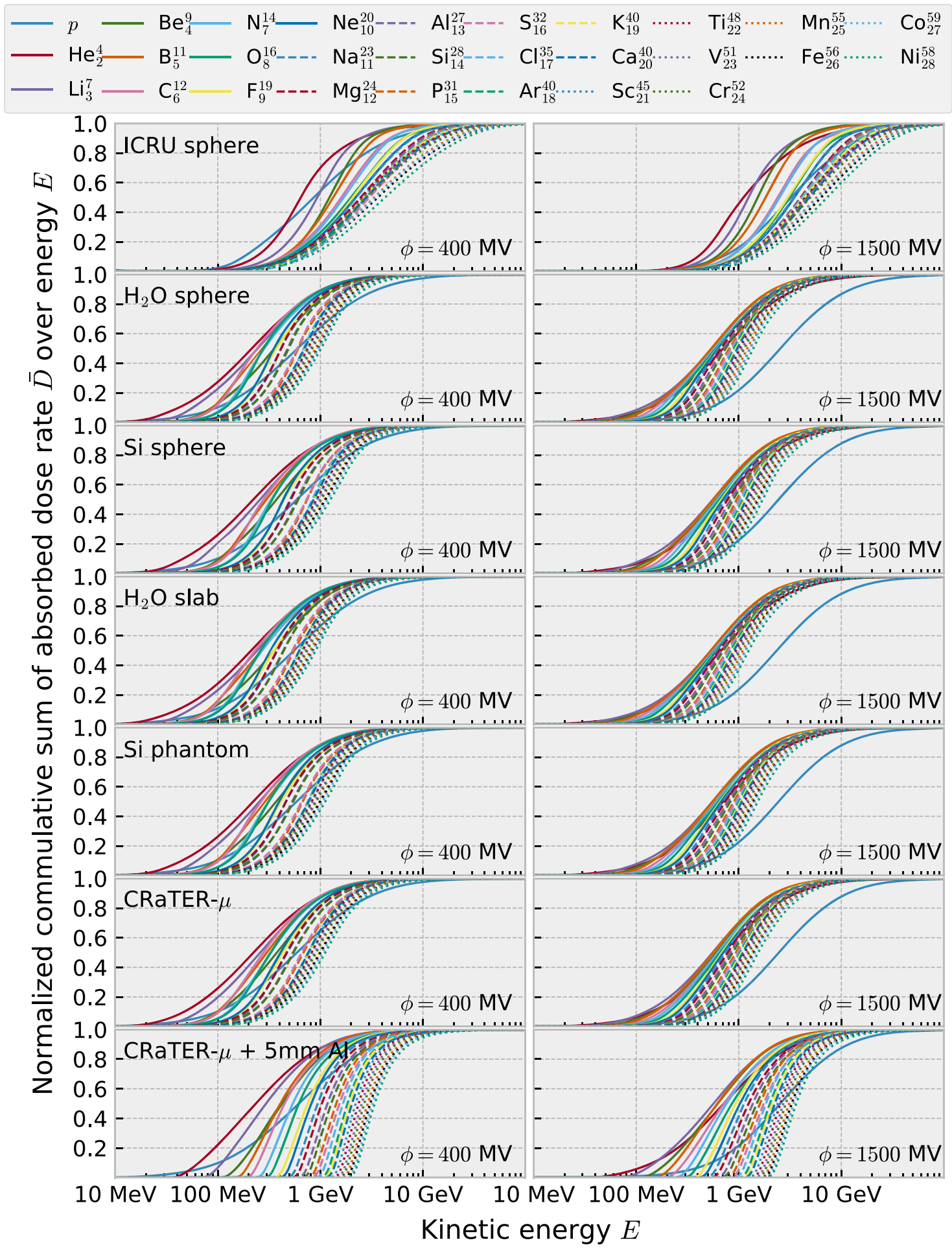

Fig. A2. The normalized cumulative sum of absorbed dose over kinetic energy has been calculated for (i) the seven detectors and (ii) the two cases of solar activity, to each corresponding a separate figure panel (look at the upper corners). Each panel contains the 28 cumulative sums corresponding to the absorbed dose rates due to different GCR nuclei. We see that the energy range between $100 \mathrm{MeV}$ and $100 \mathrm{GeV}$ is where GCR are sufficiently abundant and/or are particularly effective at depositing significant ionization energy to the detectors. 


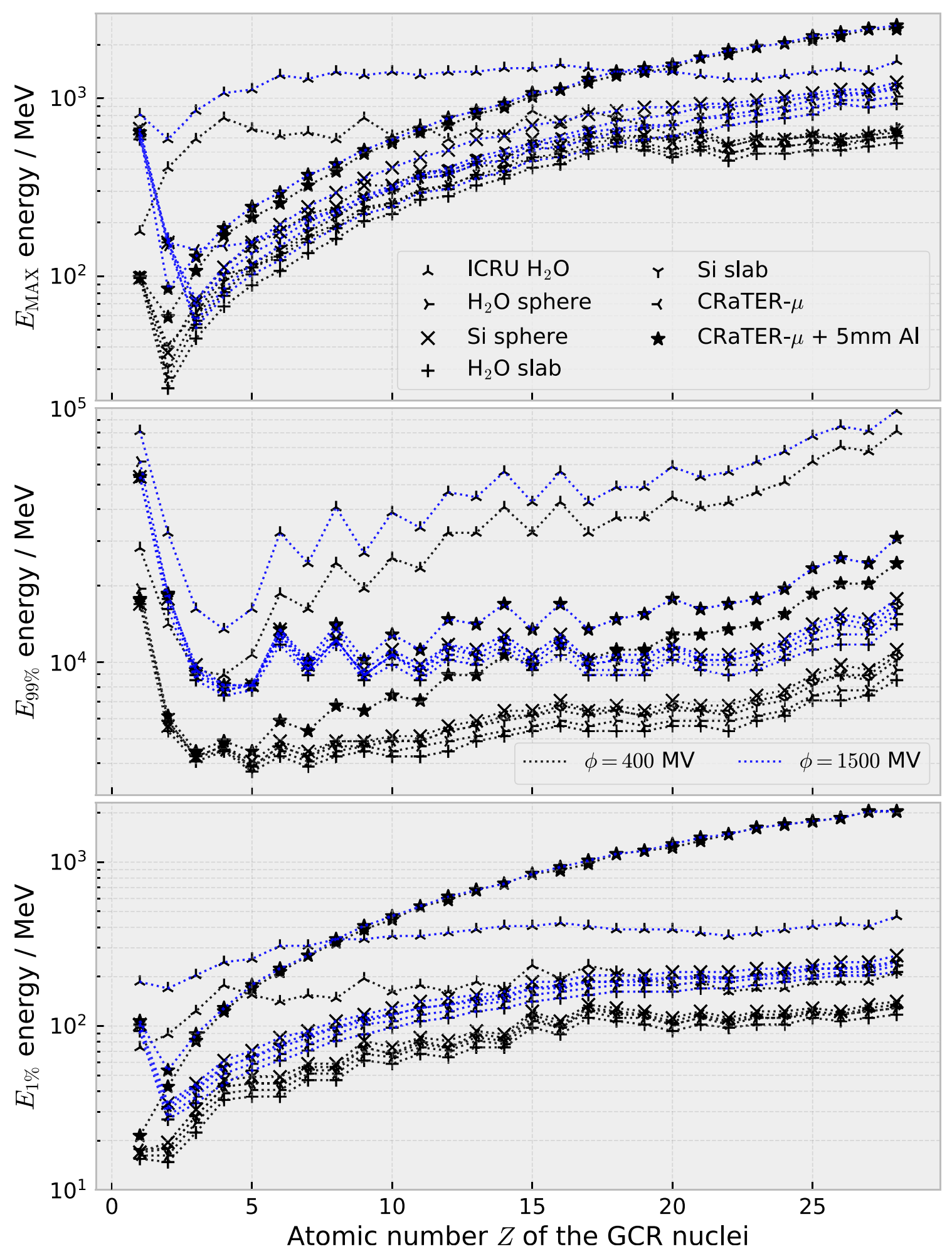

Fig. A3. In order to characterize the significant energy range for each GCR nucleus, we have calculated the most harmful kinetic energy (top panel), the kinetic energy required to achieve $99 \%$ of the net absorbed dose rate (middle panel) and the kinetic energy required to achieve $1 \%$. Each panel contains 14 scatter graphs corresponding to the seven phantoms indicated with different symbols and two levels of solar activity, indicated with two colors. For example, crosses with blue lines are for the $\mathrm{H}_{2} \mathrm{O}$ slab at solar maximum $(\phi=1500 \mathrm{MV})$. Limiting our simulation to the energy range $\left[E_{1 \%}, E_{99 \%}\right]$ would result in a underestimation ofthe absorbed dose rate of the order of $2 \%$. Important data presented here has been summarized in Table A1. 
Table A1. GCR spectrum parameters specify the energy range responsible for $98 \%$ of absorbed dose rate in each phantom. For each GCR species, as indicated in the first column, the energy range is specified for solar minimum $(\phi=400 \mathrm{MV})$ and solar maximum $(\phi=1500 \mathrm{MV})$. The CRaTER-Al column represents the microdosimeter with $5 \mathrm{~mm}$ shielding. Note that $E_{1 \%}$ is in $\mathrm{MeV}$, while $E_{99 \%}$ is in $\mathrm{GeV}$. The $E_{99 \%}$ energies have been up-rounded and the $E_{1 \%}$ have been truncated. To cover both solar minimum and maximum, the simulator should use $E_{1 \%}$ $(\phi=400 \mathrm{MV})$ and $E_{99 \%}(\phi=1500 \mathrm{MV})$.

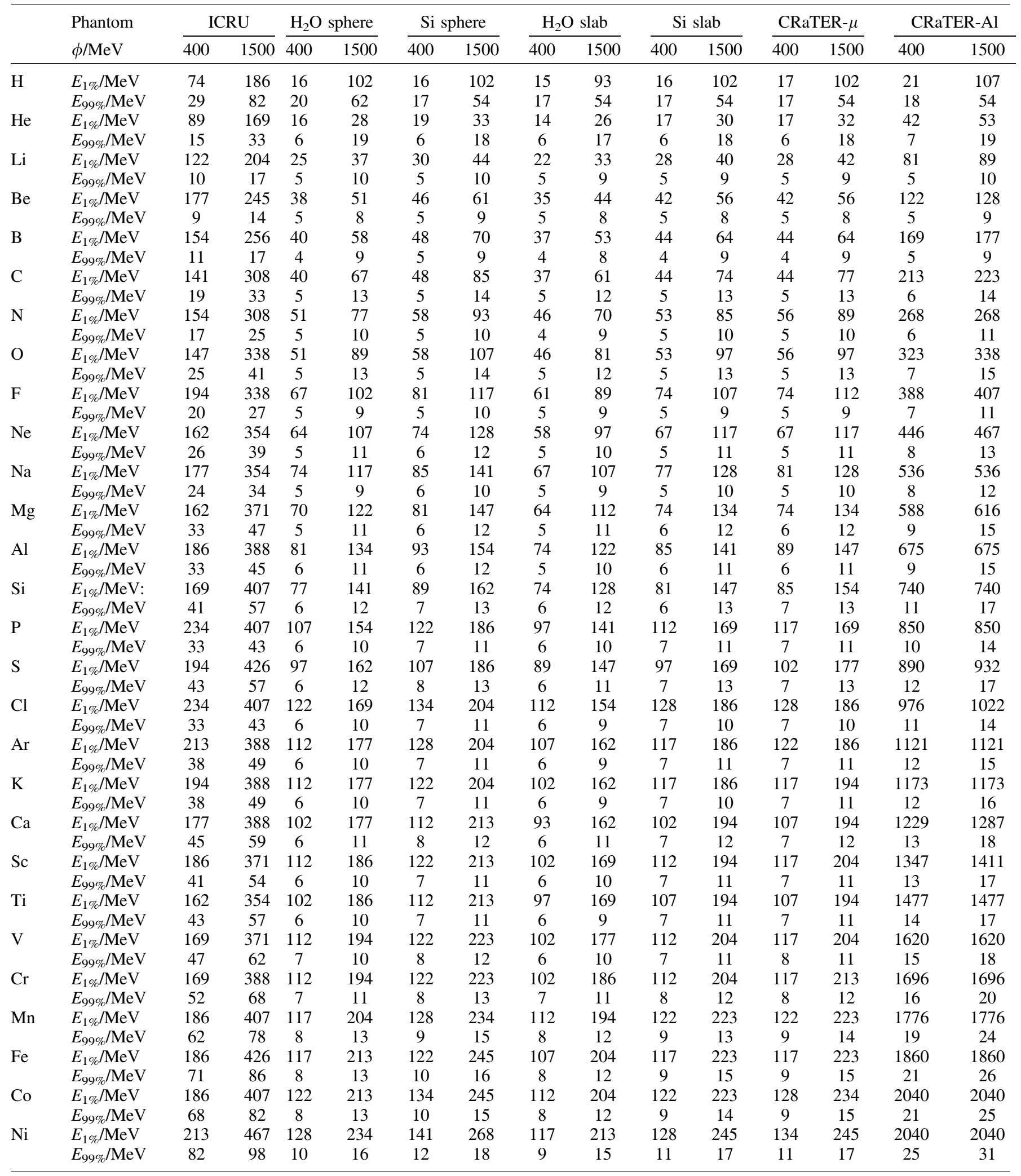


investigations. Each row represents a single detector. For example, the upper left panel corresponds to the ICRU sphere (indicated in upper left) and solar minimum with $\phi=400 \mathrm{MV}$ (indicated in lower right), while the right panel corresponds to solar maximum with $\phi=1500 \mathrm{MV}$ and so on. The so determined cumulative sum over kinetic energy delivers information about how different energies contribute to the absorbed dose rate. We can for example determine for each detector and GCR species the energy $E_{\mathrm{MAX}}$, where the dose rate increases most rapidly (shown in the top panel of Fig. A3). The middle panel shows the kinetic energy $E_{99 \%}$, at which the cumulative sums reach $99 \%$ of the dose. The so determined $E_{99 \%}$ can be used as an upper limit in simulations and in some cases, it can potentially significantly reduce the simulation time, since the time required to simulate a single particle increases significantly with energy. For completeness, in the bottom panel we show the kinetic energy $E_{1 \%}$, at which the cumulative sums reach $1 \%$ of the absorbed dose rate. Furthermore, we see that for $Z>2$ the large phantom is more sensitive to changes due to solar activity. Since the Figure 13 contains a lot of data, we have summarized for the seven phantoms an the two solar modulation cases the $E_{99 \%}$ and $E_{1 \%}$ in Table A1.

The resulting error of limiting the simulation to this energy range is an absorbed dose rate, which is underestimated by $\approx 2 \%$. While determining the relevant energy range using a $1 \%$ and $99 \%$ threshold might seem excessive since at several points we have dealt with uncertainties in the order of $\approx 20 \%$, in this case such precision is justified. The typically achieved reduction in simulation time is approximately $90 \%$ and if we were to use a higher threshold of, e.g., $10 \%$ and

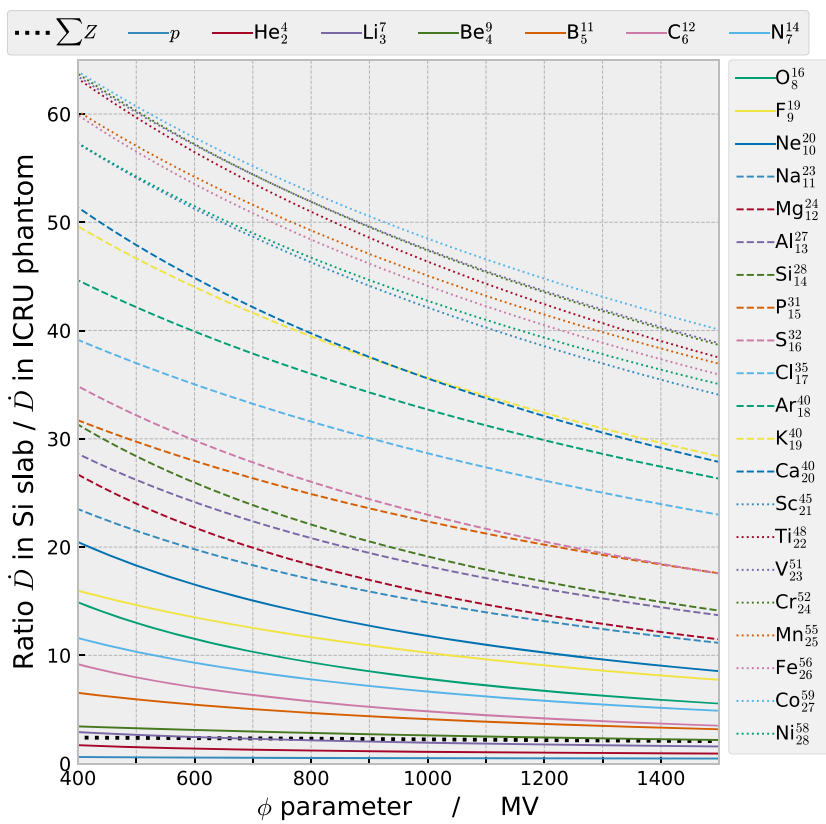

Fig. A4. Conversion factor between Si slab and the ICRU sphere as a function of the $\phi$ parameter and atomic number $Z$. The dotted black line indicates the net conversion factor.

$90 \%$, we could further narrow the energy range. However, this limitation would not result in a dramatic reduction in simulation time.

Cite this article as: Banjac S, Berger L, Burmeister S, Guo J, Heber B, et al. 2019. Galactic Cosmic Ray induced absorbed dose rate in deep space - Accounting for detector size, shape, material, as well as for the solar modulation. J. Space Weather Space Clim. 9, A14. 